Original Research Paper

\title{
Photoelectrochemical Corrosion of GaN/AIGaN-Based p-n Structures
}

\author{
${ }^{1,2}$ Alexander Usikov, ${ }^{1}$ Heikki Helava, ${ }^{3}$ Alexey Nikiforov, \\ ${ }^{4}$ Michael Puzyk, ${ }^{1}$ Boris Papchenko and ${ }^{1,5}$ Yuri Makarov \\ ${ }^{I}$ Nitride Crystals Inc., 181 E Industry Ct., Ste. B, Deer Park, NY 11729, USA \\ ${ }^{2}$ University ITMO, Kronverkskiy pr. 49, St. Petersburg 197101, Russia \\ ${ }^{3}$ Boston University, Photonics Center, 8 St. Mary's St., Boston, MA 02215, USA \\ ${ }^{4}$ Herzen University, Nab. r. Moyki 48, St. Petersburg 194186, Russia \\ ${ }^{5}$ Nitride Crystals Group Ltd., 27 Engels av., St Petersburg 194156, Russia
}

Article history

Received: 31-10-2015

Revised: $18-07-2016$

Accepted: 30-07-2016

Corresponding Author: Alexander Usikov

Nitride Crystals Inc., $181 \mathrm{E}$ Industry Ct., Ste. B, Deer Park, NY 11729, USA and

University ITMO,

Kronverkskiy pr. 49, St.

Petersburg 197101, Russia

Tel: +16312428853

Fax: +16312428906

Email: alexander.usikov@nitridecrystals.com

\begin{abstract}
Direct water photoelectrolysis using III-N materials is a promising way for hydrogen production. GaN/AlGaN based p-n structures were used as working electrodes in a photoelectrochemical process to investigate the material etching (corrosion). The structures were grown on sapphire substrates by chloride Hydride Vapor Phase Epitaxy (HVPE). First, the etching process occurs near vertically via channels associated with defects in the structure and penetrates deep into the structure. Then, the process involves etching of the n-type AlGaN barrier and n-GaN active layer in lateral direction resulting in formation of voids and cavities. The lateral etching is due to net positive charges at the $\mathrm{AlGaN} / \mathrm{GaN}$ interfaces arising because of spontaneous and piezoelectric polarization in the structure and positively charged ionized donors in the space charge region of the $\mathrm{p}-\mathrm{n}$ junction.
\end{abstract}

Keywords: Hydride Vapor Phase Epitaxy, III-N Structures, Photo-Assisted Electrochemical Process

\section{Introduction}

Hydrogen is considered a candidate as the substantial energy carrier. In the process of hydrogen energy consumption, water, as the product of hydrogen oxidation, is harmless to the environment. Water exists profusely on earth and is a resource for hydrogen production. At present, several technologies are used or being developed for hydrogen generation using solar energy directly or indirectly. They include among others thermochemical water decomposition process driven by a concentrated solar system, generation of hydrogen through biomass, hydrogen production through photosynthetic microorganisms and hydrogen production by electrolysis (Turner et al., 2008). In the latter case the electrolysis can be driven by renewable electricity and performs water decomposition electrochemically.

Direct water photoelectrolysis is a promising way for hydrogen production. In this process, a semiconductor material immersed in an aqueous solution of electrolyte allows decomposition of water into $\mathrm{H}_{2}$ and $\mathrm{O}_{2}$ gases by solar irradiation of its surface. The required energy to split water molecules is generated by sunlight absorption in the semiconductor. The solar water splitting process may be spontaneous under illumination if the electrochemical redox potentials of Oxygen Evolution Reaction (OER) and Hydrogen Evolution Reaction (HER) in an electrolyte are exceeded by the energy gap of the semiconductor. This is fulfilled for GaN-based materials (Waki et al., 2007; Ohkawa et al., 2013; Chen and Wang, 2012; Aryal et al., 2010).

Not all of the electron-hole pairs generated in the semiconductor under illumination in an electrolyte can participate in OER and (or) HER. Carrier recombination at the surface and in the bulk of the layer reduces the gas generation rate. Another process having a negative effect on the gas evolution reaction is photo-corrosion or etching of the electrode material (Wolff et al., 2005). The corrosion is an issue for n-type materials as a photoelectrode because upward band-bending at the photoelectrode/electrolyte interface promotes photogenerated holes to transfer to the electrolyte. The holes at the interface not only oxidize water (OER) but oxidize the photoelectrode. On the other hand, in p-type 
materials the band-bending promotes photo-generated electrons to transfer to the electrolyte where they participate in the HER process that almost leaves intact the surface of the photo-electrode chemically (Aryal et al., 2010; Rajeshwar, 2008; Liu et al., 2012). The p-type III$\mathrm{N}$ materials as photoelectrodes have been less investigated in the photo-electrochemical process.

A direct water photoelectrolysis feature is a relatively low photocurrent density, up to tens $\mathrm{mA} / \mathrm{cm}^{2}$, because of relatively weakly concentrated sunlight used to illuminate the photoelectrodes. The typical values of the concentration factor range from $1 \times$ to $20 \times$. Higher values of the concentration factor require a solar tracking system, resulting in electrolyte heating and necessitating an efficient heat sink. This does not comply with the concept of a simple design and low-cost usually requested from a photoelectrolysis system.

In this study we use as working electrodes $\mathrm{GaN} / \mathrm{AlGaN}$ p-n structures with p-type GaN layer on the surface to investigate the material corrosion of the photoelectrochemical process. The structure was grown on a sapphire substrate by chloride Hydride Vapor Phase Epitaxy (HVPE).

\section{Materials and Methods}

Several 8-10 $\mu$ m-thick AlGaN/GaN p-n structures were grown by chloride HVPE on c-plane 2-inch sapphire substrates in a conventional horizontal-flow reactor. The growth procedure included in-situ sapphire substrate treatment followed by multilayer structure growth consisting of $\mathrm{AlN} / \mathrm{Al}_{\mathrm{x}} \mathrm{Ga}_{1-\mathrm{x}} \mathrm{N}(\mathrm{x} \sim 0.6)$ buffer layer and 4 pairs of $\mathrm{Al}_{\mathrm{x}} \mathrm{Ga}_{1-\mathrm{x}} \mathrm{N} \quad(\mathrm{x} \sim 0.1-0.15) / \mathrm{Al}_{\mathrm{x}} \mathrm{Ga}_{1-\mathrm{x}} \mathrm{N}$ ( $x \sim 0.03-0.08$ ) Stress Control Layers (SCL) on a sapphire substrate. The $\mathrm{p}-\mathrm{n}$ structure was grown on the top of the SCL in the same run. The basic structure included a 50$100 \mathrm{~nm}$-thick $\mathrm{GaN}$ active region co-doped by $\mathrm{Zn}$ and $\mathrm{Si}$ to have emission at $420 \mathrm{~nm}$ (Usikov et al., 2003), which was sandwiched between $\mathrm{p}-$ and $\mathrm{n}-\mathrm{Al}_{\mathrm{x}} \mathrm{Ga}_{1-\mathrm{x}} \mathrm{N}$ barriers ( $\mathrm{x} \sim 0.05-0.12$ ), all grown on a 2-3 $\mu \mathrm{m}$ thick $\mathrm{n}-\mathrm{GaN}: \mathrm{Si}$ contact layer $\left(\mathrm{n} \sim 2-4 \times 10^{18} \mathrm{~cm}^{-3}\right)$. A $0.5-1 \mu \mathrm{m}$-thick ptype $\mathrm{GaN}$ contact layer doped with $\mathrm{Mg}$ covers the structure. The $\mathrm{Mg}$ doping resulted in p-type conductivity on the surface with a net acceptor concentration $\left(\mathrm{N}_{\mathrm{A}}-\mathrm{N}_{\mathrm{D}}\right)$ up to $(2-4) \times 10^{18} \mathrm{~cm}^{-3}$ as determined by $\mathrm{C}-\mathrm{V}$ characterization using $\mathrm{Hg}$ probe. Details of the structure growth and characterization can be found elsewhere (Kurin et al., 2014). Note that it was the p-type GaN layer surface that served as a photo-electrode (working electrode) in this study.

The 2-inch grown samples were immersed in a potassium hydroxide water electrolyte $(5.7$ weight $\%$ of $\mathrm{KOH}, \mathrm{pH}=14$ ) under external electrical bias of $+2.5 \mathrm{~V}$ and under solar irradiation. As a counter electrode a 2 -inch diameter $\mathrm{Ni}$ plate was positioned parallel to the working electrode at a distance of about $3 \mathrm{~cm}$. The GaN-based electrode was irradiated by a $150 \mathrm{~W}$ Xe lamp (AM 1.5 standard spectrum) with a concentration factor of $20 \times$. The irradiated area (spot size) was adjusted to 1.3 inch $(33 \mathrm{~mm})$ diameter. The electrical contact to the sample (working electrode) was made through a spring clamp with indium pads. The structures were partly immersed in the electrolyte. A segment of the structure, which was used to hang the whole wafer by the clamp, was above the electrolyte to avoid a short circuit. The whole process of photoelectrolysis took about $6 \mathrm{~min}$.

After that the samples were cleaved and their surface morphology and luminescence properties were examined in both top-view and cross-sectional configurations by high-resolution Scanning Electron Microscopy (SEM) and Cathodoluminescence (CL) spectroscopy and monochromatic CL imaging, respectively. The CL measurements were performed on a Gatan MonoCL2 system. CL spectra from three distinct areas of the samples described above were acquired by rastering a 10 $\mathrm{kV}$ electron beam over an area of $20 \times 15 \mu \mathrm{m}$. For CL spectra from cross-sections, a $8 \mathrm{nA}$ beam current was used. For CL spectra from the top surface, a slightly smaller beam current of $4 \mathrm{nA}$ was used to mitigate electron beam induced charging on the top surface.

\section{Results}

After the experiment, three areas can be seen on the structure surface by naked eye: (1) Area 1 that was not immersed into $\mathrm{KOH},(2)$ Area 2 that was immersed into $\mathrm{KOH}$ but not illuminated by the Xe lamp and (3) Area 3 that was immersed into $\mathrm{KOH}$ and illuminated by Xe lamp. Area 3 on the surface of the structure appears to be dimmer than its surroundings.

Fig. 1a shows SEM images of cross sectional views of the same sample taken at Area 2 on the surface. All layers of the structure can be distinguished. The structure total thickness is about $9.5 \mu \mathrm{m}$. Note that in the experiment $\mathrm{a}+2.5 \mathrm{~V}$ bias was applied to the $\mathrm{p}$-type top layer of the structure from external power source, i.e., the structure was a photo anode. During the experiment the measured value of photocurrent was found to decrease from 4.3 to $2.2 \mathrm{~mA}\left(0.5-0.25 \mathrm{~mA} / \mathrm{cm}^{2}\right)$. Total thickness of the p-type layers is about $0.7 \mu \mathrm{m}$. No peculiarities that can be attributed to the photoelecrochemical etching are observed. It seems that the value of photocurrent passing through Area 2 (the area that was immersed into the electrolyte but not illuminated by the $\mathrm{Xe}$ lamp) was too small to demonstrate a noticeable etching.

Fig. 1b shows a SEM image of the cross-sectional view of Area 3 of the same structure. All layers of the structure are the same as in Fig. 1a. However two rows 
of micro-voids can be ascribed to the photoelecrochemical etching. The position of the voids in the stack of layers in the structure makes it possible to assume that these voids are formed in the GaN active layer and in the underlying $\mathrm{n}-\mathrm{AlGaN}$ barrier layer. The micro-voids can spread laterally and overlap leading to the formation of a large-size cavity. No apparent changes in the composition or elemental distribution of the constituent elements before and after etching were observed in the films as verified by results of energydispersive spectrometry.

Fig. 2 shows the large-size cavity that formed after the photoelctrolysis process performed on another AlGaN/GaN p-n structure but having higher photocurrent of $5.6-9.7 \mathrm{~mA}\left(0.65-1.1 \mathrm{~mA} / \mathrm{cm}^{2}\right)$ under the same illumination. The large-size cavity may result in delamination of the p-layers from the structure. Fig. 3 shows a scanning micrograph of surface morphology in Area 3 of the sample in Fig. 1. Pits and large open cavities on the surface are attributed to the photoelectrochemical process. The pits density is about $2 \times 10^{8}$ $\mathrm{cm}^{-2}$ and it varies over the sample surface up to (12) $\times 10^{9} \mathrm{~cm}^{-2}$ in the most defective areas near the microcracks. The CL spectra acquired from the sample surface have a weak emission at about $360 \mathrm{~nm}$ and a dominant broad peak centered around $420 \mathrm{~nm}$ as shown in Fig. 4a. The strongest luminescence was measured from the area illuminated by the Xe lamp (Area 3) where the density of nonradiative defects was likely decreased as a result of the photo-electrochemical etching. The coarser surface morphology in Area 3 may also improve the light extraction efficiency.

The dominant emission can be ascribed to the active layer located at a depth of 0.5-0.6 microns from the surface. Although the $10 \mathrm{kV}$ beam penetration is limited to about 0.4 microns in depth (Yacobi and Holt, 1986), the electron beam spreading and various surface irregularities, such as pits and micro-cracks, allow beam electrons to reach active region located underneath the $p$ type contact layers and creates electron-hole pairs within the active layer. Their subsequent recombination results in an enhanced CL signal observed from the structure surface. A 1-3 $\mu \mathrm{m}$ thick Mg-doped p-type GaN layer grown in a separate run had a PL peak emission near 440 $\mathrm{nm}$. Broad CL spectra overlap the emission from the $\mathrm{p}$ GaN contact layer in Fig. 4.

To further elucidate the origin of the luminescence peaks, the CL spectra were acquired from cross-sections from the three distinct areas defined above. The spectra are shown in Fig. 4b. A strong broad emission from the active region at $414-420 \mathrm{~nm}$ is complemented by a strong signal from n-GaN contact layer at around 360 $\mathrm{nm}$. Emission in the range of 324 to $331 \mathrm{~nm}$ could be ascribed to the AlGaN barriers. The rastered area included the substrate for reference purposes, for example, for a proper identification of the substrate-SCL layer interface in the monochromatic CL mapping.

The cross-sectional monochromatic CL maps acquired at the corresponding wavelengths of 324-331 $\mathrm{nm}$ and $420 \mathrm{~nm}$ for both samples are shown in Fig. 5. The maps at 324-331 $\mathrm{nm}$ and at $360 \mathrm{~nm}$ for both samples are very similar as they indicate that the corresponding luminescence emissions are coming from the AlGaN layers and from the GaN barriers in the bulk of the structure, respectively. The $420 \mathrm{~nm}$ emission corresponds to the GaN active region and is mainly localized within about a micron thick region near the top surface and correlates with subsurface void formation and top layer delamination in the area etched and illuminated by the Xe lamp (Area 3).

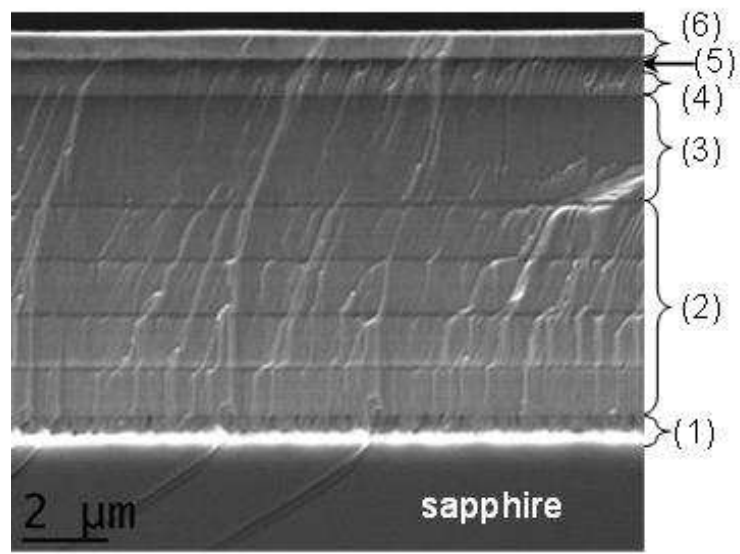

(a)

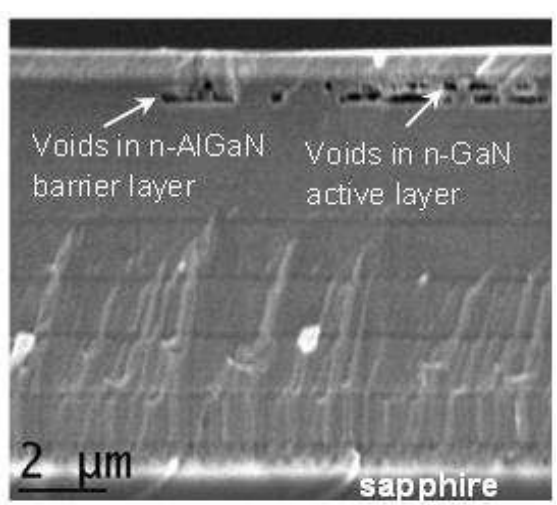

(b)

Fig. 1. Cross-sectional SEM image of different areas of the AlGaN/GaN p-n structure after photo-electrolysis.(a) Area 2. No peculiarities related to the photo-electrochemical etching are observed. (1) AlN/AlGaN buffer, $0.65 \mu \mathrm{m}$; (2) AlGaN/AlGaN four pairs, $4.97 \mu \mathrm{m}$; (3) n-GaN:Si contact layer, $\sim 2.5 \mu \mathrm{m}$; (4) n-AlGaN barrier layer, $\sim 0.5 \mu \mathrm{m}$; (5) GaN:(Zn+Si) active layer, $\sim 0.2 \mu \mathrm{m}$; (6) $\mathrm{p}-\mathrm{GaN}$ contact $+\mathrm{p}-\mathrm{AlGaN}$ barrier layers, $\sim 0.66 \mu \mathrm{m}$. (b) Area 3. Two rows of voids observed in the $\mathrm{n}-\mathrm{GaN}$ active layer and in the n-AlGaN barrier lay are attributed to photo-electrochemical etching 


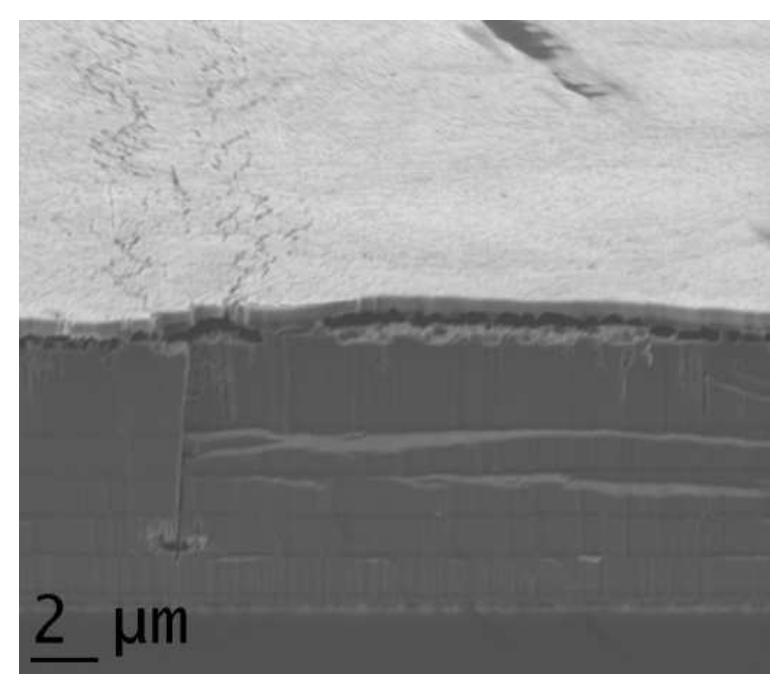

Fig. 2. Cross-sectional SEM image of Area 3 of another AlGaN/GaN p-n structure. Overlapped micro-voids that formed under higher photocurrent than in Fig. 2 sample off the p-layers from the structure created a large-size cavity that may delaminate or peel

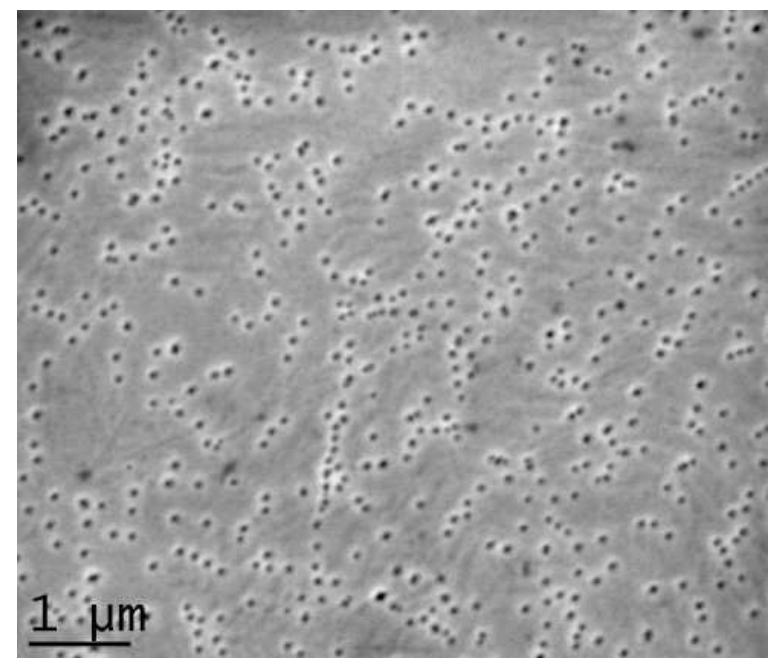

Fig. 3. SEM images of surface morphology after photo-electrochenical etching of AlGaN/GaN p-n structure in Fig. 1. Pits density is about $2 \times 10^{8} \mathrm{~cm}^{-2}$

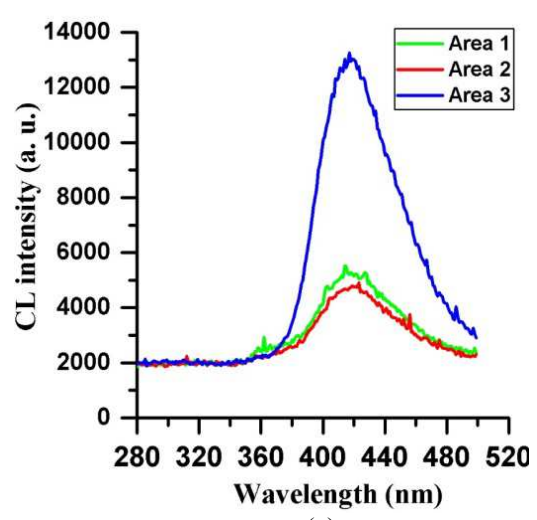

(a)

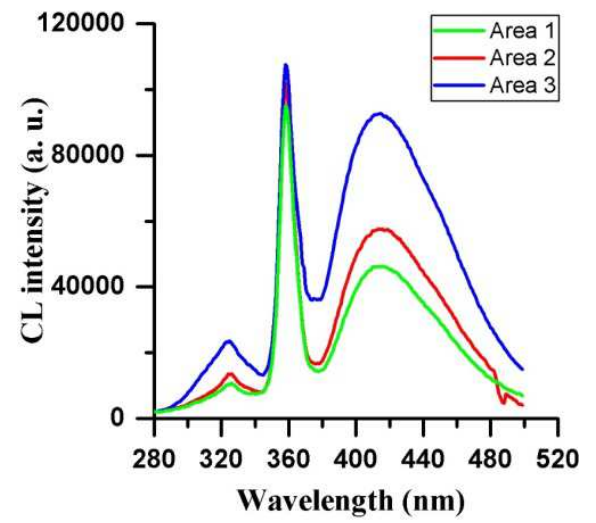

(b)

Fig. 4. CL spectra acquired from the sample surface (a) and from a cross section of the sample (b). Emission from the active region at $\sim 420 \mathrm{~nm}$ is observed in both configurations 


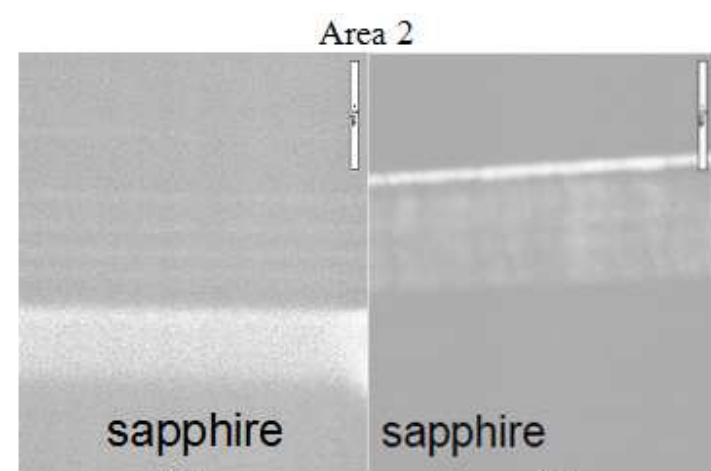

(a) (b)

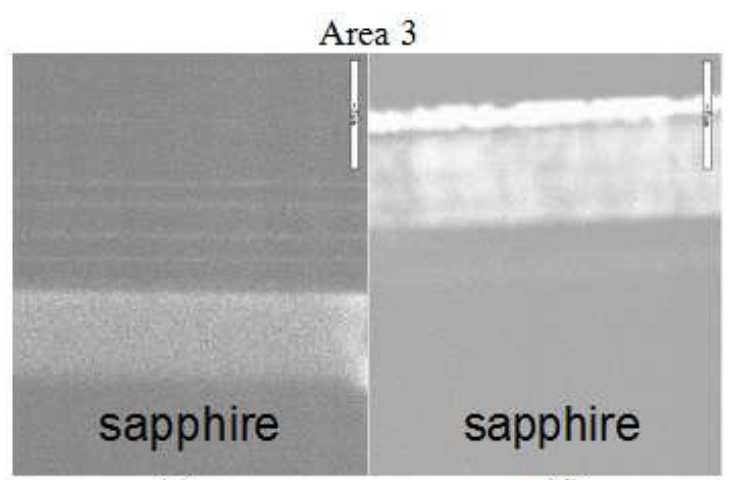

(c) (d)

Fig. 5. Cross-sectional monochromatic CL maps after photo-electrochemical etching acquired at two areas at 324-331 nm (a, c); and $420 \mathrm{~nm}$ (b. d) for sample in Fig. 1. The scale bar is $5 \mu \mathrm{m}$

\section{Discussion}

It was observed that photocurrent dropped in the first minute and become more or less stable later on at the experiment. Such behavior is typical for the electrochemical process with GaN-based electrodes (Aryal et al., 2010; Ohkawa et al., 2013) and can be associated with effectively decreasing of the electrode area that is contacting the electrolyte mainly due to the electrode corrosion and a production of gas microbubblers $\left(\mathrm{O}_{2}, \mathrm{H}_{2}, \mathrm{~N}_{2}\right)$ that are result of the electrochemical process and can passivate the electrode surface. Obviously, the photoelectrochemical etching follows paths of the photo-current flow. The current flow paths in III-N GaN/AlGaN or GaN/InGaN materials, especially at low current, are nonuniform and can be associated with shunt conductivity in an extended defect system of grain boundaries, threading dislocations, local regions with irregular alloy composition enriched by metallic atoms ( $\mathrm{Ga}$ or $\mathrm{In}$ ) and others extended defects (Shmidt et al., 2015).

Voids observed in the SEM images in Figs. 2-3 suggest that the process of their formation proceeds in two stages. During the first stage, the etching process starts via channels (or shunts) in the extended defect system of the structure penetrating the p-type layers all the way into the underlying n-type layers. The shunts could be threading defects, grain boundaries and microcracks attributed to mosaic structure of the structures asgrown. During the second stage, the etching on the ntype AlGaN barrier and GaN active layer proceeds in a lateral direction resulting in the formation of voids and cavities underneath the p-type layers. Although the ptype layers of the structure remained unetched, the top surface exhibited a lot of pits.

The UV portion of the illumination light gets effectively absorbed in the p-GaN contact layer (at a depth of about 0.2-0.3 um from the surface) leading to the formation of excited electron-hole pairs. These pairs can be separated further by either the internal electric field of the space-charge region in the $p$-n junction of the structure when they drift towards it or by the specific band-bending at the $\mathrm{p}-\mathrm{GaN}$ layer/electrolyte interface. The potential barrier at the $p-n$ junction is higher than the bend-bending at the surface of the p-GaN layer and the separation of the excited electron-hole pairs could be further affected by $p-n$ junction resulting in the generation of a photocurrent. The photo-generated carriers may participate in two competitive processes in the structure employed as an anode and immersed into an electrolyte under irradiation: (1) Oxygen Evolution Reaction (OER) and (2) photo-etching of $\mathrm{GaN}$ and GaN-based materials. However, because of a too thick p-GaN layer (0.5-0.6 $\mu \mathrm{m}$ thick) the excited electron-hole pairs recombine mostly at the structure surface and in the p-layer before being separated by the $\mathrm{p}-\mathrm{n}$ junction producing as a result a relatively low photocurrent. It appears that relatively low photocurrent flows non-uniformly through the structure selecting paths with higher conductivity that are threading defects and micro cracks.

We speculate that photo-generated holes could assist in oxidative decomposition of $\mathrm{p}-\mathrm{GaN}$, primarily in the vicinity of the threading defects forming dangling bonds of $\mathrm{Al}^{3+}$ and $\mathrm{Ga}^{3+}$ atoms. Hydroxide ions $\left(\mathrm{OH}^{-}\right)$from the electrolyte would then attack the $\mathrm{Al}^{3+}$ and $\mathrm{Ga}^{3+}$ dangling bonds and form hydroxides $\mathrm{Al}(\mathrm{OH})_{3}$ and $\mathrm{Ga}(\mathrm{OH})_{3}$ (Zhuang and Edgar, 2005; Macht et al., 2005). Dissolution of the hydroxides would lead to the formation of soluble $\mathrm{Al}_{2} \mathrm{O}_{3}$ and $\mathrm{Ga}_{2} \mathrm{O}_{3}$. Continuous formation and dissolution of the oxides in the solution would further simulate the etching process.

The etching process may move deeper into the layers via the threading defects. The Mg-doped p-type GaN and $\mathrm{AlGaN}$ layers have ionized $\mathrm{Mg}^{-}$acceptors and may screen partly the positive charge of the dangling bonds preventing the etching of the p-type layers but still allowing the etching to proceed along the threading 
defects. Indeed we did not observe any appreciable etching of the top p-layers. However, some micro-cracks and numerous pits associated with etching were observed on the top surface as can be seen in Fig. 3. It is likely that these pits are associated with dislocations.

The step involving lateral etching in Figs. 1-2 can be explained considering effects resulting in a positive charge at layer interfaces. It is know that spontaneous and piezoelectric polarization between $\mathrm{AlGaN} / \mathrm{GaN}$ layers in the structure results in a formation of a net positive charge at their interface (Mishra et al., 2002). Fig. 6 shows a net positive charge at the AlGaN/GaN interface in the AlGaN layer caused by the sum of the net spontaneous polarization and piezoelectric polarization between the $\mathrm{AlGaN}$ and GaN layers in the structure growing along the $c$-direction (Mishra et al., 2002). $Q_{\pi}$, AlGaN includes the contribution of spontaneous and piezo-electric contributions for AlGaN layer and $Q_{\pi}, \mathrm{GaN}$ is the only spontaneous polarization for the GaN layer as it has been assumed to be relaxed due to a much larger thickness than that of the AlGaN layer.

The positive $+Q_{\pi}$ charge at the bottom side of the thick GaN layer in Fig. 6a has a negligibly small effect on the charges at the $\mathrm{AlGaN} / \mathrm{GaN}$ interface due to a presence of large number of electrons, traps and defects between the interface and the bottom portion of the thick underlying $\mathrm{GaN}$ layer. The sheet charge due to polarization in the $\mathrm{AlGaN}$ layer surface and at the interface is balanced also. To keep neutrality, the positive charge at the interface is compensated by electron accumulation forming a two Dimensional Electron Gas (2DEG) close to the interface (Mishra et al., 2002), the source of electrons in the 2DEG is the surface states (Ibbetson et al., 2000).

The structures in this study had a stack of doped GaN and $\mathrm{AlGaN}$ layers at the top (i.e., the n-GaN active layer was sandwiched between $\mathrm{n}$ - and $\mathrm{p}$-AlGaN barrier layers) that influences the net positive charge compare to that depicted in Fig. 5. First of all, negatively charged ionized acceptors $\left(\mathrm{Mg}^{-}\right)$in the p-AlGaN layer may screen positive polarization charge at the $\mathrm{p}-\mathrm{AlGaN}$ barrier layer/n-GaN active layer interface. In addition, a thin $n$ $\mathrm{GaN}$ active layer is not fully relaxed and may have additional positive piezoelectric polarization charge at the $\mathrm{n}-\mathrm{GaN}$ active layer/n-AlGaN barrier layer interface.

Then, n-AlGaN barrier also has a positive piezoelectric polarization charge at the interface with $\mathrm{n}-\mathrm{GaN}$ contact layer beneath (Fig. 1a for reference to the structure design). Note also that all thin AlGaN layers having higher composition in the AlGaN/AlGaN four-pairs structure, beneath the nGaN contact layer, have a net positive polarization charge. However these layers are located deep in the structure, further away from the structure surface. Only two thin n-GaN active and n-AlGaN barrier layers are near the p-type layers and have positive piezoelectric polarization charge. An additional positive charge at the layer interfaces can be connected with positively charged ionized donors, which are $\mathrm{Si}+$ presumably, in a space charge region of the $\mathrm{p}(\mathrm{AlGaN})-\mathrm{n}(\mathrm{GaN})$ junction. This positive charge is located in the $\mathrm{n}-\mathrm{GaN}$ active region too. Our separate experiments on photoelectrolysis with GaN layers only demonstrated porous-like etching of 5-6 $\mu \mathrm{m}$ thick n$\mathrm{GaN}$ layers through the layer to the substrate whereas in $\mathrm{p}-\mathrm{n} \mathrm{GaN}$ structures having a $\mathrm{p}-\mathrm{GaN}$ layer on the top the etching process was terminated on the $p-n$ junction.

The net positive charge at the GaN/AlGaN n-n and pn interfaces promotes $\mathrm{OH}^{-}$ions from the electrolyte to form $\mathrm{Ga}(\mathrm{OH})_{3}$ and (or) $\mathrm{Al}(\mathrm{OH})_{3}$ hydroxides and the nitrogen vacancy sites and continue the etching process along the positive charge position forming lateral voids and large-size cavities.

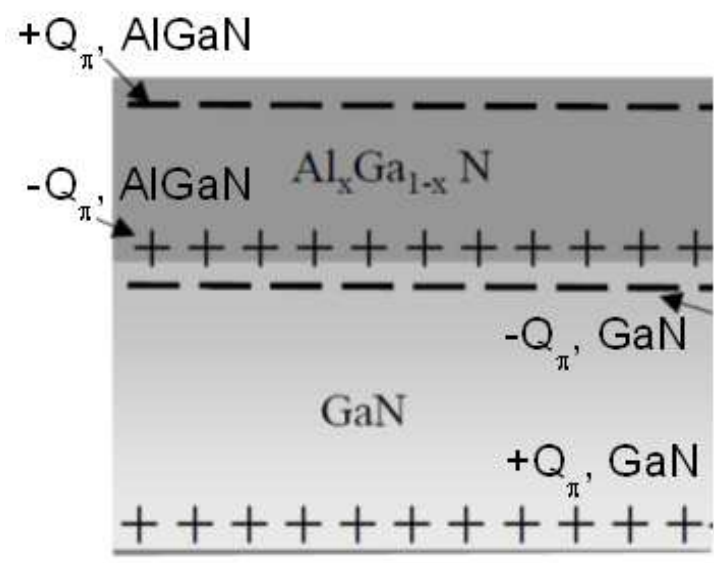

(a)

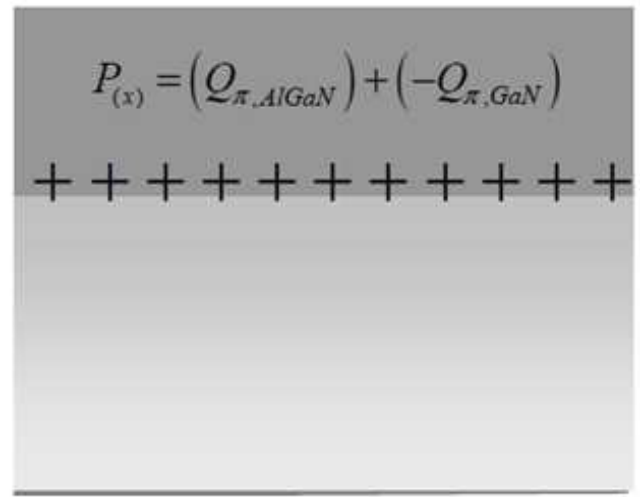

(b)

Fig. 6. CL Polarization charges in an $\mathrm{AlGaN} / \mathrm{GaN}$ structure. (a) Charge polarization in individual layers of the structure (b) a net positive charge at the $\mathrm{AlGaN} / \mathrm{GaN}$ interface in the AlGaN layer (Mishra et al., 2002) 
When the etching process via threading defects reaches a layer with positive net charge it changes the etching direction from vertical to lateral presumably because of a stronger charge at the layer interface than that in the channel associated with threading defects and dangling bonds. When large threading defects, for example, large cracks are encountered the etching process may penetrate deeper into the structure as shown in Fig. 3b. However, the n-type AlGaN layer located at a depth from the surface switches the etching process from a vertical to a lateral direction due to a larger positive piezoelectric polarization charge at the layer interfaces.

Recent investigations of degradation mechanisms for similar HVPE-grown AlGaN/GaN p-n LED structures using multifractal analysis indeed demonstrated the existence of conductive paths (shunts) localized at threading defects (dislocations) (Shmidt et al., 2015). In our structures, the shunts are leakage paths for photocurrier flow that are responsible for non-uniform current spreading, diminishing the gas evolution reaction and decreasing the efficiency of direct water photoelectrolysis. Low-defect GaN-based working photoelectrodes are important for the photoelectrolysis process by focusing on gas generation but not etching.

\section{Conclusion}

Two stages of photoelectrochemical etching under conditions of light illumination and external power source were observed in a GaN/AlGaN p-n structure having p-type layers on the top. During the first stage, the etching process occurs near vertically via channels associated with defects in the structure and penetrates deep into the structure to n-type layers passing p-type layers. The p-type layers of the structure remain unetched in the process. During the second stage, the process involves etching of the n-type AlGaN barrier and $\mathrm{GaN}$ active layer in a lateral direction resulting in the formation of voids and cavities beneath the p-type layers. The lateral etching is likely due to net positive charges at the AlGaN/GaN interfaces arising because of spontaneous and piezoelectric polarization in the structure and positively charged ionized donors in the space charge region of the $\mathrm{p}-\mathrm{n}$ junction.

\section{Acknowledgement}

Work at University ITMO was supported by the Ministry of Education and Science of Russian Federation (grant agreement 14.575.21.0054, unique identifier of research activities is RFMEFI57514X0054).

\section{Author Contributions}

Alexander Usikov: Participation in the photoelctrolysis experiments, writing of the manuscript and give final approval of the version to be submitted and any revised version.

Heikki Helava: Design and select of the GaN/AlGaN p-n structures used in the experiments, reviewing of the manuscript for technical data and intellectual content.

Alexey Nikiforov: Acquisition of SEM and CL data and drafting of the manuscript.

Michael Puzyk: Participation in the photoelctrolysis experiments, analysis and interpretation of data.

Boris Papchenko: Design the work plan, organized the study and drafting of the manuscript.

Yuri Makarov: Growth of the GaN/AlGaN p-n structures by HVPE and Coordinated of the post-growth chatracterization of the structures.

\section{Ethics}

This article is original and contains unpublished material. The corresponding author confirms that all of the other authors have read and approved the manuscript and no ethical issues involved.

\section{References}

Aryal, K., B.N. Pantha, J. Li, J.Y. Lin and H.X. Jiang, 2010. Hydrogen generation by solar water splitting using p-InGaN photoelectrochemical cells. Applied Phys. Lett., 96: 052110-1-052110-3.

DOI: $10.1063 / 1.3304786$

Chen, S. and L.W. Wang, 2012 Thermodynamic oxidation and reduction potentials of photocatalytic semiconductors in aqueous solution. Chem. Mater., 24: 3659-3666. DOI: $10.1021 / \mathrm{cm} 302533 \mathrm{~s}$

Ibbetson, J.P., P.T. Fini, K.D. Ness, S.P. DenBaars and J.S. Speck et al., 2000. Polarization effects, surface states and the source of electrons in $\mathrm{AlGaN} / \mathrm{GaN}$ heterostructure field effect transistors. Applied Phys. Lett., 77: 250-252. DOI: 10.1063/1.126940

Kurin, S., A. Antipov, I. Barash, A. Roenkov and A. Usikov et al., 2014. Characterization of HVPEgrown UV LED heterostructures. Phys. Status Solidi, 11: 813-816. DOI: 10.1002/pssc.201300459.

Liu, S.Y., J.K. Sheu, M.L. Lee, Y.C. Lin and S.J. Tu et al., 2012. Immersed finger-type indium tin oxide ohmic contacts on $\mathrm{p}$-GaNphotoelectrodes for photoelectrochemical hydrogen generation. Opt. Express, 20: A190. DOI: 10.1364/OE.20.00A190

Macht, L., J.J. Kelly, J.L. Weyher, A. Grzegorczyk and P.K. Larsen, 2005. An electrochemical study of photoetching of heteroepitaxial GaN: Kinetics and morphology. J. Crystal Growth, 273: 347-356.

DOI: $10.1016 /$ j.jcrysgro.2004.09.029

Mishra, U.K., P. Parikh and Y.F. Wu, 2002. AlGaN/GaN HEMTs: An overview of device operation and applications. Proc. IEEE, 90: 1022-1031.

DOI: $10.1109 /$ JPROC.2002.1021567 
Ohkawa, K., W. Ohara, D. Uchida and M. Deura, 2013. Highly stable GaN photocatalyst for producing $\mathrm{H}_{2}$ gas from water. Japanese J. Applied Phys., 52: 08JH04-08JH04. DOI: 10.7567/JJAP.52.08JH04

Rajeshwar, K., 2008. Hydrogen Generation from Irradiated Semiconductor-Liquid Interfaces. In: Solar Hydrogen Generation: Toward a Renewable Energy Future, Rajeshwar, K., R. McConnell and S. Licht (Eds.), Springer New York, ISBN: 978038772809-4, pp; 167-228.

Shmidt, N., E. Shabunina, A. Usikov, A. Chernyakov and S. Kurin et al., 2015. Peculiarities of defect generation under injection current in LEDs based on A3N nanostructure. Phys. Status Solidi, 12: 1136-1139. DOI: $10.1002 /$ pssc. 201400218

Turner, J., G. Sverdrup, M.K. Mann, P.C. Maness and B. Kroposki et al., 2008. Renewable hydrogen production. Int. J. Energy Res., 32: 379-407. DOI: $10.1002 /$ er. 1372

Usikov, A.S., D.V. Tsvetkov, M.A. Mastro, A.I. Pechnikov and V.A. Soukhoveev et al., 2003. Indium-free violet LEDs grown by HVPE. Phys. Status Solidi, 0: 2265-2269. DOI: 10.1002/pssc.200303521
Waki, I., D. Cohen, R. Lal, U. Mishra and S.P. Den Baars et al., 2007. Direct water photoelectrolysis with patterned n-GaN. Applied Phys. Lett., 91: 093519-093519.

DOI: $10.1063 / 1.2769393$

Wolff, N., M. Rapp and T. Rotter, 2005. Electrochemical etching and CV-profiling of GaN. Phys. Status Solidi, 2: 990-993. DOI: 10.1002/pssc.200460607

Yacobi, B.G. and D.B. Holt, 1986. Cathodoluminescence scanning electron microscopy of semiconductors. J. Applied Phys., 59: R1-R24. DOI: $10.1063 / 1.336491$

Zhuang, D. and J.H. Edgar, 2005. Wet etching of GaN, AlN and SiC: A review. Mater. Sci. Eng.: R Rep., 48: 1-46. DOI: 10.1016/j.mser.2004.11.002 